\title{
Evolution of the Dark Matter Distribution with 3-D Weak Lensing
}

\section{J. Bacon ${ }^{1} \dagger$, A. N. Taylor ${ }^{1}$, M. L. Brown ${ }^{1}$, M. E. Gray ${ }^{2}$, C. Wolf ${ }^{3}$, K. Meisenheimer ${ }^{4}$, S. Dye ${ }^{5}$, L. Wisotzki ${ }^{6}$, A. Borch $^{4}$, M. Kleinheinrich ${ }^{4}$}

\footnotetext{
${ }^{1}$ Institute for Astronomy, University of Edinburgh, Blackford Hill, Edinburgh, EH9 3HJ, U. K.

${ }^{2}$ School of Physics and Astronomy, University of Nottingham, University Park, Nottingham, NG7 2RD, U.K.

${ }^{3}$ Department of Physics, University of Oxford, Keble Road, Oxford OX1 3RH, U.K.

${ }^{4}$ Max-Planck-Institut für Astronomie, Königstuhl 17, D-69117, Heidelberg, Germany

${ }^{5}$ Astrophysics Group, Blackett Laboratory, Imperial College, Prince Consort Road, London SW7 2BW, U.K.

${ }^{6}$ Astrophysikalisches Institut Potsdam, An der Sternwarte 16, D-14482 Potsdam, Germany
}

\begin{abstract}
We present a direct detection of the growth of large-scale structure, using weak gravitational lensing and photometric redshift data from the COMBO-17 survey. Deep $R$-band imaging of two $0.5 \times 0.5$ square degree fields is used to provide shear estimates for over 52000 galaxies; these are combined with photometric redshift estimates from our 17 band survey, in order to obtain a 3-D shear field. We discuss how theoretical models for evolving matter power spectra and correlation functions cab be used to find a best fit to this 3-D shear field. We present the detection of the evolution of the power, and measurements of the rate of evolution for $0<z<1$. We discuss future refinements which will improve the accuracy with which the effect can be measured.
\end{abstract}

\section{Introduction}

It is clear that the phenomenon of gravitational lensing has an intimate relationship with redshift information. Consider for instance the weak gravitational lensing distortion matrix:

$$
\Psi_{i j}=\frac{\partial \delta \theta}{\partial \theta_{j}}=\int_{0}^{z_{\text {source }}} d z g(z) \frac{\partial^{2} \Phi}{\partial \theta_{i} \partial \theta_{j}}
$$

Here we see that $\Psi_{i j}$ depends upon redshift in several ways. First, the upper limit of the line-of-sight integral depends upon the redshift of the source galaxy one is observing; $\Psi_{i j}$ will grow as a function of distance to source galaxies, which will be related to the depth of the survey. Secondly, the geometrical weight function $g(z)$ is a varying function of the redshift of lenses along the line of sight. Finally, $\Psi_{i j}$ depends upon the gravitational potential at all points along the line of sight; this gravitational potential may itself, at a given scale, be evolving with redshift.

It is clear, therefore, that if we measure the lensing potential (or equally, shear or convergence) as a function of redshift, we will obtain information about both the geometry of the Universe as a function of redshift, and the evolution of the distribution of matter. Various studies have investigated the utility of this effect (e.g. Hu 1999, Taylor 2001,

$\dagger$ Email: djb@roe.ac.uk 


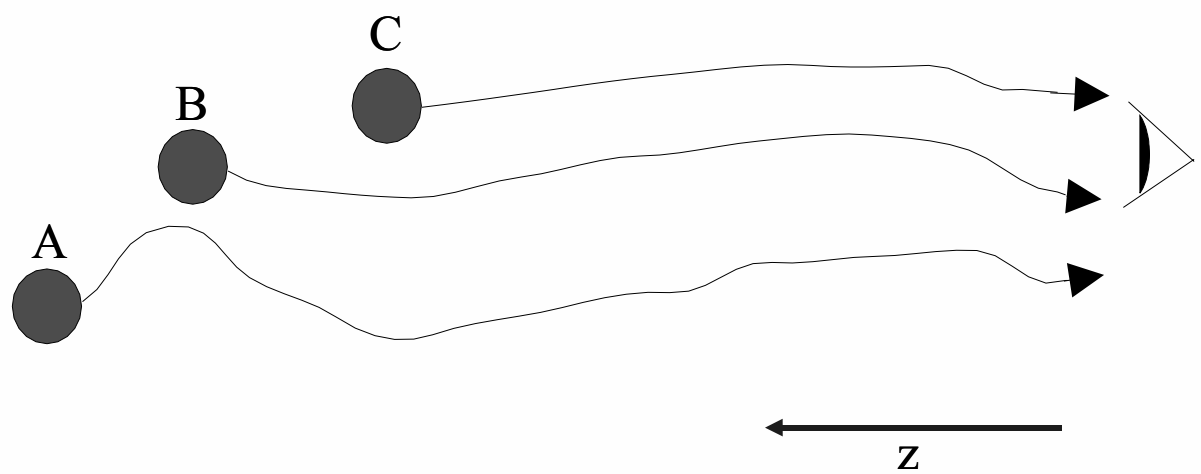

Figure 1. Concept of 3-D lensing. The fact that light rays are distorted between A and B, or B and $\mathrm{C}$, allows us to differentially assess the distribution of dark matter in these redshift ranges.

Wittman et al 2001, Huterer 2002, Hu \& Keeton 2002, Heavens 2003, Jain \& Taylor 2003, Bacon \& Taylor 2003, Simon, King \& Schneider 2004, Bacon et al 2004).

A simple way of understanding the concept of 3-D lensing is shown in Figure 1. Consider a set of galaxies (for simplicity, with circular pre-shear images) A, B and C along almost the same line of sight, but with different redshifts. The light from the galaxies will travel along a similar trajectory in the foreground which they share; i.e. the light from each galaxy will be bent by the foreground mass concentrations. However, the light from galaxy B will be further distorted when traveling the extra path between $\mathrm{B}$ and $\mathrm{C}$, due to additional mass concentrations in this region; the light from galaxy $\mathrm{A}$ will be distorted further still when traveling between $\mathrm{A}$ and $\mathrm{B}$. By measuring the different distortions of galaxies $\mathrm{A}, \mathrm{B}$ and $\mathrm{C}$, we can differentially infer the evolution of mass concentrations along this line of sight.

Another way of conceptualising the methodology of 3 -D lensing is to move from discussing a 2-dimensional shear field $\left(\gamma_{1}(x, y), \gamma_{2}(x, y)\right)$ to a 3 -dimensional shear field $\left(\gamma_{1}(x, y, z), \gamma_{2}(x, y, z)\right)$, i.e. we are now considering the (2 component) distortion which we would observe upon a galaxy image, given that the source galaxy is at some point in the 3-dimensional Universe.

Several applications can be derived using this general combination of lensing with redshift information. Firstly, one can make 3-D maps of the dark matter distribution (Taylor et al 2004; c.f. Taylor in this volume). This could lead to an approach for finding clusters directly in 3-D by detecting their potential wells; it also allows comparison of the 3 -D dark matter distribution with the 3-D distribution of number density of galaxies, or luminosity density in the Universe.

Secondly, one can measure various statistics of the 3-D shear field in order to compare with the predicted statistics from cosmological models, hence measuring important cosmological parameters such as $\sigma_{8}, \Omega_{m}$ or $w$, the equation of state of the dark energy (e.g. Simon et al 2004, Bacon et al 2004; c.f. King, Heavens in this volume).

A further, alternative approach is to invert the 3-D shear field in order to directly measure the evolution of the dark matter distribution. It is this approach which we address here. 


\section{Data}

For our initial exploration of the evolution of the power spectrum, we will use data from the COMBO-17 survey (Wolf et al 2001). This survey contains $50.5 \times 0.5 \mathrm{deg}^{2}$ fields, with observations taken in 5 broad band filters and 12 medium band filters in order to obtain exceptionally accurate photometric redshifts for member galaxies. Here we study two of these fields (S11 and CDFS) which have full photometric redshift catalogues. A further field, A901/2 which is useful for 3-D mapping (Taylor et al 2004), is excluded from this analysis on account of it being selected to include a supercluster; it is therefore too exceptional a line of sight for cosmic evolution statistics.

We use the shear catalogue for these COMBO-17 fields obtained by Brown et al (2003), measured from deep $R$ band imaging with 0.8 " median seeing. We also use the photometric redshift catalogue obtained by Wolf et al (2001); this provides us with a redshift accuracy (for galaxies which actually are assigned a redshift) of $\Delta_{z}=0.05$ for $0<z<1$ for our shear sample.

We combine the shear and photometric redshift catalogues to obtain a final, 3-D shear catalogue containing $\left(x, y, z, \gamma_{1}, \gamma_{2}\right)$ information for each galaxy. In total, we have 52139 usable galaxies for our shear analysis, 23102 of which have photometric redshifts. For the galaxies without a defined photometric redshift, we will optionally assign a median redshift $z=0.9 \pm 0.1$; this is obtained by extrapolating the median redshift - median magnitude relation (c.f. Brown et al 2003).

\section{Measuring the Evolution}

We measure the evolution of the dark matter distribution using shear correlation functions. 2-dimensional versions of these are familiar from standard cosmic shear analyses, i.e. $\left\langle\gamma_{i}(0) \gamma_{j}(\theta)\right\rangle$. Predictions for the values of the 2-dimensional shear correlation functions as a function of galaxy separation are often calculated from a model evolving matter power spectrum. This power spectrum, $P_{\delta}$, is projected to provide a prediction of the 2-D shear correlation function $C(\theta)$ (e.g. Bartelmann \& Schneider 2001):

$$
C(\theta)=\int_{0}^{\infty} l d l J_{0}(l \theta) \int_{0}^{w_{H}} F(w) P_{\delta}(l / w, w)
$$

where $F$ is a function of the radial comoving distance $w$.

This is a correlation function which finds an average distortion correlation for source galaxies found at all redshifts accessible to a survey. Instead of doing this, we can calculate a correlation function directly between galaxies with particular redshifts. This does not mean that we simply obtain $C(\theta, z)$; this would mean a correlation function where we looked at the correlation of galaxies within redshift shells. Rather, we can calculate $C\left(\theta, z_{1}, z_{2}\right)$, i.e. the correlation function for galaxies separated by an angle and at different redshifts. This corresponds to replacing $F(w)$ above with a slightly more complex function of the particular source redshifts, i.e. $F\left(w, w_{1}, w_{2}\right)$ (c.f. Bacon et al 2004). As can be seen from equation (3.1), the correlation function grows with redshift in a way that is specific to the particular evolution of the model power spectrum (c.f. equation (1.1)); so by measuring the correlation from data as a function of $\theta, z_{1}$ and $z_{2}$ we can obtain direct information on the evolution of the power spectrum of matter.

The way we achieve this in practice is to use a simple model for the evolving power spectrum:

$$
P_{\delta}(k, z)=A k^{-\alpha} e^{-s z}
$$



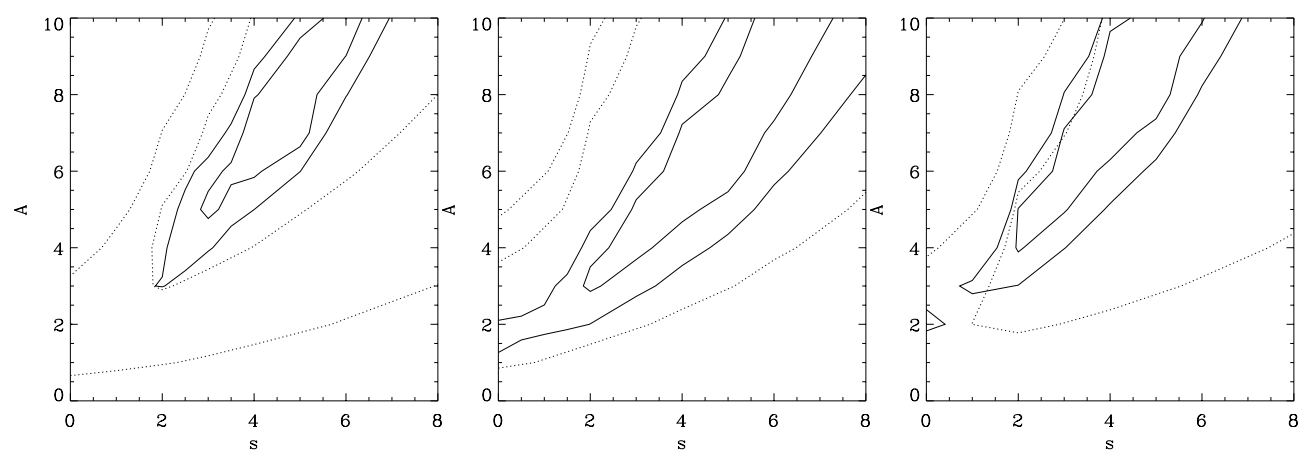

Figure 2. Plot showing maximum likelihood contours for $A$ and $s$. Solid lines show 1 and $2 \sigma$ contours with assigned medium redshift for background population; dotted lines show contours for only galaxies with known photometric redshift. Left panel shows result for both fields combined; middle panel shows result for CDFS, and right panel shows result for S11.

This is a simplified power spectrum, separable in radial and tangential directions; this is found to be a suitable approximation in the measurable regime by examining the shape of $C\left(\theta, z_{1}, z_{2}\right)$. Equivalently, we find that $\alpha=1.2$ is a good fit to $\Lambda$ CDM predictions for the correlation function throughout $1^{\prime}$ to $30^{\prime}$ and $0<z<1$. It is useful to note the physical meaning of $A$ and $s$ : $A$ measures the present-day amplitude of the power spectrum, while $s$ is a measure of the rate of evolution of the dark matter distribution.

From the above equation we calculate a model shear correlation function, $C\left(\theta, z_{1}, z_{2}\right)$, fixing the geometry to $\Lambda \mathrm{CDM}$ (i.e. $\Omega_{\Lambda}=0.7, \Omega_{m}=0.3$ are used for calculating cosmological distance measures). By doing this, we will be investigating the way that the 3-D shear data depends specifically on growth of power, rather than on evolution and geometry combined. We then carry out a maximum likelihood fit to the data, varying $A$ and $s$.

The results of this process can be seen in Figure 2 (c.f. Bacon et al 2004). Note that a no-evolution hypothesis, i.e. $s=0$, is significantly excluded if we include a median redshift for our background sample $(s=0$ excluded at the $7.7 \sigma$ level with the mild prior that the present-day power spectrum has $\left.\sigma_{8}>0.4\right)$; we are therefore detecting the evolution of the dark matter distribution. Note that there is a degeneracy between $A$ and $s$; if the present day amplitude of the power spectrum is made larger, the rate of evolution is also constrained to be larger. Note also that the range of slope and amplitude allowed varies from field to field.

We can calculate from these $(A, s)$ plots the permitted range for the power spectrum itself as a function of redshift (Bacon et al 2004). This is shown in Figure 3. Note that $\Lambda \mathrm{CDM}$ growth is permitted within $2 \sigma$ permitted models at all redshifts, but equivalently we could state that it is not particularly favoured by the current data. A larger dataset will be required to overcome this sample variance, and further work will be carried out to examine if any systematic uncertainties remain in the evolution estimate.

Nevertheless, we see that the data strongly favour an evolving power spectrum, which is a fundamental prediction of cosmology. This method therefore shows promise; with a larger survey, it should truly allow the precise determination of the evolution of the dark matter distribution. This could furnish us with a test of gravity and/or the behaviour of dark matter. It could be combined with studies of evolution of the baryon distribution in order to understand the combined dark matter-baryon evolution. Finally, since the particular pattern of structure growth is sensitive to the equation of state of the Dark 

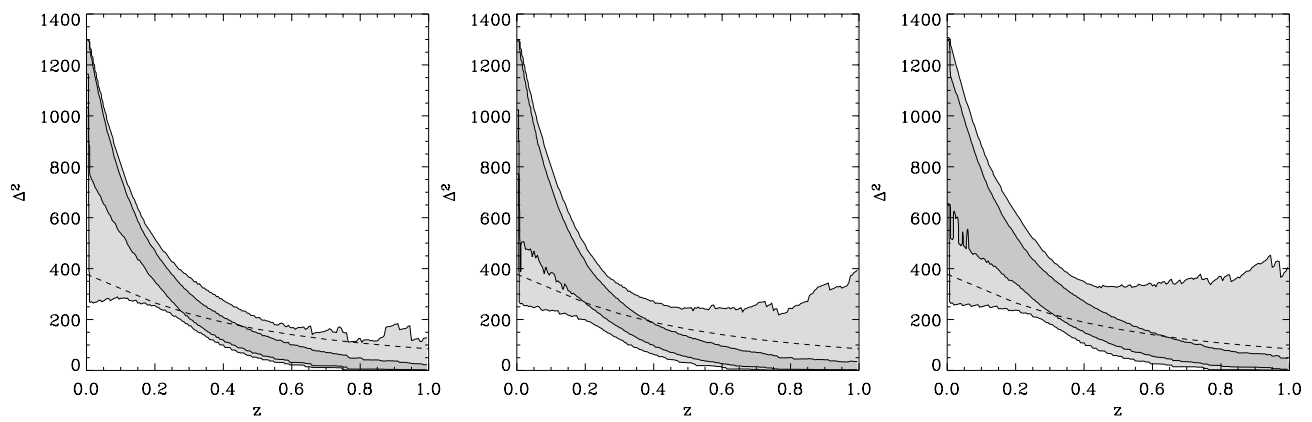

Figure 3. Plot showing allowed range for the power spectrum as a function of redshift. Here we have plotted a slice at $k=14 \mathrm{Mpc}^{-1}$. The dark grey area is the $1 \sigma$ allowed region for the power spectrum; light grey shows $2 \sigma$. As above, the panels are for the combined, CDFS and S11 power spectra.

Energy, this type of 3-D lensing analysis could afford us measurements of this important quantity.

\section{Refinements}

The above results demonstrate the value of combining shear and photometric redshifts. However, they also indicate that several refinements to the process are highly desirable and should be implemented in future studies.

Firstly, it is vital that the statistical significance of these results is improved. The error bars on the redshift evolution of the power spectrum are large, and could be reduced by sampling a much larger area. However, there is also an uncertainty that is not quoted above: that of sample variance, the fact that any given line of sight will have a set of fluctuating structures along it which do not represent the mean evolution along all lines of sight. We currently have a high sample variance due to only having two fields at present; further data will allow us to overcome the Poisson noise arising from having only a small number of structures along a few lines of sight.

Secondly, it should be noted that the current analysis is limited by the number of galaxies that have usable photometric redshift estimates. As seen on Figure 2, our results are greatly improved if we assign a median redshift to the faint $(R>23)$ background galaxy sample. This is understandable; it is of great value in constraining the growth of the dark matter distribution to know the impact of lensing on the high redshift objects. A complete redshift catalogue to a higher magnitude threshold (e.g. $R=25$ ) would transform the analysis, but will be very difficult to come by in practice. Further work is required to optimise a survey strategy for measuring the evolution of the dark matter distribution, given the difficulty of obtaining precise photometric redshifts for faint objects.

Other important extensions include simulations of the effect and measurement method, in order to test the reliability of these measurements, especially concerning the impact of redshift uncertainties. A further extension will be to combine 3-D shear correlation functions with 3-D flexion correlation functions (c.f. Goldberg \& Bacon 04) in order to better constrain the dark matter evolution on small scales. 


\section{Conclusions}

This paper has provided a brief introduction to some key aspects of 3-D weak lensing, i.e. using shear estimates together with photometric redshifts. We have seen that the lensing effect is a function of redshift via geometric factors (i.e. distances) and the evolution of the density. The evolution of the dark matter distribution can therefore be observed using 3-D lensing.

We have shown how the growth of structure can be detected in practice, using the COMBO-17 survey as a useful example; this survey has been subjected to a thorough shear analysis, and has accurate photometric redshift estimates for approximately half the galaxies in the survey. We have calculated model 3-D shear correlation functions representing an evolving dark matter distribution, and have fitted these to our data as a function of amplitude of the power spectrum at the current epoch and rate of growth of the power.

In this fashion we detect the growth of structure on the COMBO-17 fields. We find an evolution rate that is compatible with $\Lambda \mathrm{CDM}$, and see variation from field to field, as we would expect from sample variance. We have also directly calculated the allowed amplitude for the matter power spectrum as a function of redshift.

This initial study has therefore demonstrated that 3-D lensing should be a useful tool for measuring the growth of structure, and comparing evolution of dark matter and baryonic components. Similar maximum likelihood methodology will also afford increasingly precise measurements of cosmological parameters such as $\Omega_{m}, \sigma_{8}$ and the equation of state of the Dark Energy, w (c.f. Bacon et al 2004). The next step for study in this area is to examine the optimal approach to 3-D lensing surveys, obtain larger surveys, and push the bounds of measuring photometric redshifts for faint galaxy samples.

\section{Acknowledgements}

DJB is supported by a PPARC Postdoctoral Fellowship. We would like to thank Alan Heavens and Hans-Walter Rix for extremely useful discussions.

\section{References}

Bacon D. J., Taylor A. N., Brown M. L., Gray M. E., Wolf C., Meisenheimer K., Dye S., Wisotzki L., Borch A., Kleinheinrich M. 2004, submitted to MNRAS, astro-ph/0403384.

Bacon D. J., Taylor A. N. 2003, MNRAS 344, 1307.

Bartelmann M., Schneider P. 2001, Phys. Rep. 340, 291.

Brown M. L., Taylor A. N., Bacon D. J., Gray M. E., Dye S., Meisenheimer K., Wolf C. 2003, MNRAS 341, 100.

Goldberg D. M., Bacon D. J. 2004, submitted to ApJ, astro-ph/0406376.

Heavens A. 2003, MNRAS 343, 1327.

Hu W. 1999, ApJL 522, 21.

Hu W., Keeton C. R. 2002, PhysRevD 66, 063506.

Huterer D. 2002, PhysRevD 65, 063001.

Jain B., Taylor A. 2003, PhysRevLett 91, 141302.

Simon P., King L. J., Schneider P. 2004, A\&A 417, 873.

Taylor A. N. 2001, submitted to Phys Rev Lett, astro-ph/0111605.

Taylor A. N., Bacon D. J., Gray M. E., Wolf C., Meisenheimer K., Dye S., Borch A., Kleinheinrich M., Kovacs Z., Wisotzki L. 2004, accepted by MNRAS, astro-ph/0402095.

Wittman D., Tyson J. A., Margoniner V. E., Cohen J. G., Dell'Antonio I. P. 2001, ApJ 557, 89.

Wolf C., Meisenheimer K. \& Roeser H.-J. 2001, A\& A 365, 660. 